\title{
Évaluation de l'activité anxiolytique de la 4-phenyl-1,5-benzodiazepin-2-one
}

\author{
P. M. KANYONGA ${ }^{1 *}$, M. Y. A. FAOUZI ${ }^{2}$, A. ZELLOU ${ }^{2}$, \\ M. E. ESSASSI ${ }^{1}$ et Y. CHERRAH ${ }^{2}$ \\ ${ }^{1}$ Laboratoire de Chimie Organique Hétérocyclique, Pôle de Compétences Pharmacochimie, \\ Faculté des Sciences- Agdal, B.P :1014, Avenue : Ibn Battouta / Raba, Maroc. \\ ${ }^{2}$ Laboratoire de Pharmacologie et Toxicologie, Faculté de Médecine et Pharmacie, Rabat - Instituts, Maroc. \\ *Auteur correspondant, E-mail : manuelekanyonga@yahoo.fr
}

\section{RESUME}

Les effets pharmacologiques de la 4-phenyl-1,5-benzodiazepin-2-one ont été examinés chez des souris par des tests comportementaux classiques (Test de planche à trous, Test de labyrinthe et Test de la tige tournante) et comparés par rapport aux produits dont le potentiel anxiogenique ou anxiolytique sont connus. L'analyse de nos résultats a montré que la 4-phenyl-1,5-benzodiazepin-2-one et le diazépam ont augmenté le temps passé dans les bras ouverts, tandis que la picrotoxin a entraîné la diminution de ce temps. Considérant les résultats de l'évaluation de l'activité locomotrice, du comportement exploratoire et du comportement des animaux sur la tige tournante, le diazépam et la 4-phenyl-1,5-benzodiazepin-2-one ont influencé de façon significative ces derniers par rapport à la picrotoxin et au véhicule. Ces données suggèrent que la 4-phenyl-1,5benzodiazepin-2-one possède une activité anxiolytique.

(C) 2009 International Formulae Group. All rights reserved.

Mots clés: 4-phenyl-1,5-benzodiazepin-2-one, activité anxiolytique, test de planche à trous, test de labyrinthe, test de la tige roulante.

\section{INTRODUCTION}

Les benzodiazépines sont des médicaments psychotropes les plus largement consommés dans le monde en raison de leur activité anxiolytique, anticonvulsivant et myorelaxant (Beaulieu, 2006; Tosti et al., 2007). Toutefois, des effets indésirables comme la dépendance physique, la tolérance, l'ataxie et le trouble de mémoire ont été associés à leur utilisation. La plupart des benzodiazépines utilisées en clinique thérapeutique sont issus de la classe de la 1,4benzodiazépine (Fernandes et al., 1996; Zangrossi et al., 1999), puisque plusieurs produits ont été obtenus par l'introduction des substituants en différentes positions de cette dernière, et voir même par modification de la structure de l'anneau. Les effets anxiolytique et anticonvulsivant de 1,5-benzodiazépine (clobazam) ont été décrits (De Sarro et al., 1992; Remy, 1994; Widlocher, 2002; Perlemuter, 2008). Toutefois, on sait beaucoup moins sur les effets de substituants sur la 1,5-benzodiazépine par rapport au groupe de la 1,4-benzodiazepine, c'est ainsi que la classe de 1,5-benzodiazepine est davantage explorée, dans le but de rechercher des nouveaux produits capables d'interagir avec les récepteurs des benzodiazépines, et de mettre au point des produits ayant moins d'effets secondaires (Wolfman et al., 1994; Van Gerven et al., 1997), mais la synthèse de ces molécules utilise des méthodes nécessitant la mise en jeu de réactifs onéreux, voire même dangereux (Yasumatsu et al., 1994; Kirkiacharian, 1996; Graham et Depovere, 2002). 
C'est ainsi que, nous nous sommes fixés pour objectif de synthétiser la 4-phenyl1,5-benzodiazépin-2-one en utilisant des réactifs usuels et d'explorer son activité anxiolytique par comparaison avec des substances de référence. Ce qui permettra de dire si la 4-phenyl-1,5-benzodiazépin-2-one possède la propriété anxiolytique reconnue aux benzodiazépines.

\section{MATERIEL ET METHODES Produit testé}

La 4-phényl-1,5-benzodiazépin-2-one a été synthétisée par condensation de l'ophénylenediamine avec le benzoylacétate d'éthyle dans le xylène pendant une heure (Essassi et al., 1987; Zellou et al., 1998a), suivant le mode opératoire ci-dessous:

- Mettre dans un ballon de $250 \mathrm{ml}, 0,01$ mole d'ortho-phénylène-diamine et 0,011 mole de benzoylacètate d'éthyle dans $100 \mathrm{ml}$ de xylène, porter à reflux pendant 1 heure et refroidir. Après l'obtention d'un précipité, filtrer et laver avec de l'éthanol, puis sécher et on obtient une poudre de couleur blanche.

Et, sa structure (Figure 1) a été déterminée sur base des paramètres spectrales et des données physicochimiques (Tableau 1): Les points de fusion non corrigés ont été mesurés à l'aide du banc chauffant Köffler. Les spectres de résonance magnétique nucléaire $\left({ }^{1} \mathrm{H},{ }^{13} \mathrm{C}\right)$ ont été enregistrés sur un appareil AVANCE 300 Bruker opérant à 300 $\mathrm{MHz}$, en solution dans le chloroforme deutérié. Les déplacements chimiques sont donnés en ppm par rapport à la référence interne TMS. Les spectres de masse ont été effectués par impact électronique à l'aide de l'appareil VARIAN MAT 311A. Les spectres IR ont été enregistrés en film de $\mathrm{KBr}$, sur un spectromètre IR-TF VERTEX 70 entre 600 et $4000 \mathrm{~cm}^{-1}$.

\section{Produits de référence}

- Diazépam,

- Picrotoxin (Sigma Chemical Co., St. Louis, MO, USA).

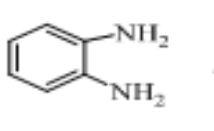

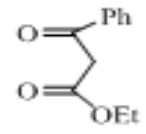

2<smiles>O=C1CNc2ccccc2N=C(c2ccccc2)C1</smiles>

3

Figure 1: Schéma de synthèse de la 4-phényl-1,5-benzodiazépin-2-one.

Tableau 1 : Paramètres d'identification de la 4-phényl-1,5-benzodiazépin-2-one.

\begin{tabular}{|c|c|c|}
\hline Rendement & Point de fusion & Données spectrales \\
\hline \multirow{3}{*}{$90 \%$} & \multirow{3}{*}{$\begin{array}{l}198-200{ }^{\circ} \mathrm{C} \\
\text { (Ethanol) }\end{array}$} & $\begin{array}{l}\text { * Spectre } \mathrm{RMN}^{1} \mathrm{H}\left(\mathrm{CDCl}_{3}\right): 3,4 \mathrm{ppm}\left(2 \mathrm{H}, \mathrm{S}, \mathrm{CH}_{2}\right) \\
7,20-7,90 \mathrm{ppm}(9 \mathrm{H} \text { aromatiques, m); } 9,37 \mathrm{ppm} \\
(1 \mathrm{H}, \mathrm{s}, \mathrm{NH}) ;\end{array}$ \\
\hline & & $\begin{array}{l}\text { * Spectre RMN 13C : } 39,79 \mathrm{ppm}(\mathrm{CH} 2) ; 121,82- \\
130,98 \mathrm{ppm}(\mathrm{CH} \text { aromatique }) ; 129,14 \mathrm{ppm}(\mathrm{C} \\
\text { aromatique }) ; 137,62-139,91 \mathrm{ppm}(\mathrm{C} \\
\text { aromatique }) ; 158,64 \mathrm{ppm}(=\mathrm{N}) ; 167,80 \mathrm{ppm} \\
(\mathrm{C}=\mathrm{O}) ; \\
\text { * Spectre IR: } \mathrm{v}_{\mathrm{C}=\mathrm{O}}: 1670 \mathrm{Cm}^{-1} ;\end{array}$ \\
\hline & & $*$ Spectre de masse $: \mathrm{M}^{+}(\mathrm{m} / \mathrm{z})=236$ \\
\hline
\end{tabular}




\section{Animaux}

L'étude est effectuée sur des souris Swiss adultes males, provenant de l'élevage du Laboratoire de Pharmacologie de la Faculté de Médecine et de Pharmacie de Rabat, dont le poids se situe entre 20 et 30 g, ils étaient maintenus dans les mêmes conditions de température $\left(25 \pm 2{ }^{\circ} \mathrm{C}\right)$, l'humidité relative située entre $50 \pm 5 \%$, et l'accès à l'eau et aux aliments à volonté. (Broustail, 1967 ; Laroche et Rousselet, 1990). L'utilisation des animaux a été faite en conformité avec les guides d'utilisation des animaux de Laboratoire (Ernest, 1993; Anonyme, 1996).

Les produits ont été administrés par voie intraperitoneale sous forme d'une suspension dans la gomme arabique à $10 \%$, en raison de $0,5 \mathrm{ml}$ pour $20 \mathrm{~g}$ du poids corporel.

\section{Etude de l'activité anxiolytique}

L'activité anxiolytique de la 4-phényl1,5-benzodiazépin-2-one a été évaluée sur base des tests comportementaux, et les résultats étaient comparés aux produits de référence.

Le test de labyrinthe (Lister, 1987; Ferreri, 2004 ; Leboyer, 2005), permet de mesurer l'anxiété (anxiété liée à la confrontation avec une situation nouvelle). Pour ce faire, nous avons utilisé un dispositif en forme de croix, surélevé de $50 \mathrm{~cm}$ audessus du sol. Il comprend quatre bras de $50 \mathrm{~cm}$ de longueur et de $10 \mathrm{~cm}$ de largeur. Deux bras opposés sont fermés par des parois verticales de $45 \mathrm{~cm}$ de hauteur, les deux autres étant ouverts sur le vide et, de ce fait, anxiogènes. L'animal est placé au centre de la croix et peut accéder librement à l'ensemble des quatre bras. Pendant 10 minutes, on observe le comportement de l'animal dans le dispositif. On mesure le nombre d'entrées dans les bras ouverts et dans les bras fermés, ainsi que le temps passé dans chacun de ces bras. Un faible nombre d'entrées et un temps court passé dans les bras ouverts sont considérés comme des indices d'anxiété.

Le test de planche à trou (Lister, 1987; Cherrah et al., 2007): permet d'évaluer le comportement exploratoire et l'activité locomotrice des animaux. Pour ce faire, on utilise une planche de $40 \times 40 \mathrm{~cm}$ et de $1,8 \mathrm{~cm}$ d'épaisseur, percé de 16 trous de $3 \mathrm{~cm}$ de diamètre, régulièrement espacés. La souris est déposée au centre de la planche et on compte le nombre de fois où la souris plonge la tête dans un des trous au bout de 1, 2, 3, 4 et 5 minutes et on calcule la moyenne de trous explorés pendant 5 minutes, et le nombre de zones traversées par l'animal avec toutes les pattes.

Les doses suivantes ont été utilisées : le diazépam (0,1-1 mg/kg), la 4-phényl-1,5benzodiazépin-2-one $(50,100,200 \mathrm{mg} / \mathrm{kg})$ et la picrotoxin $(0,2-2 \mathrm{mg} / \mathrm{kg})$.

Le test de la tige tournant (Tsuda et al., 1996 ; Cherrah et al., 2007), permet d'évaluer l'effet de la coordination des mouvements après administration des produits à tester, 25 minutes après administration du véhicule et des produits (le diazépam $1 \mathrm{mg} / \mathrm{kg}$ et la 4 phényl-1,5-benzodiazépin-2-one : 50, 100 et $200 \mathrm{mg} / \mathrm{kg}$ ), les animaux étaient placés dans l'appareil et on a enregistré le nombre de chutes et le temps moyen de séjour sur la tige avant la chute (maximum $300 \mathrm{~s}$ ). Les animaux ayant fait l'objet de cette étude ont été retenus sur base du temps passé sur la tige lors d'essai avant le test; ainsi seuls les animaux ayant séjourné une minute ou plus sur la tige ont été sélectionnés.

\section{Analyse statistique}

L'analyse statistique des données a été effectuée par l'ANOVA, ainsi que le test de Student-Newman-Keuls. Les valeurs sont considérées comme significatives à $\mathrm{P}<0,05$.

\section{RESULTATS}

Test de Labyrinthe: la 4-phényl-1,5benzodiazépin-2-one, comme le diazépam, a augmenté le temps passé dans les bras ouverts $[\mathrm{F}(5,98)=3,26, \quad \mathrm{p}<0,01]$, alors que la picrotoxin a diminué le temps passé dans les bras ouverts par les animaux (Figures 2, 3 et 4).

Le Test de la planche à trous montre que la picrotoxin $(2 \mathrm{mg} / \mathrm{kg})$ a provoqué une réduction du nombre de trous explorés [F $(3,59)=10,09, \quad \mathrm{p}<0,001]$ et l'activité locomotrice des animaux; en revanche, le diazépam a augmenté le nombre de trous explorés à la dose de $0,1 \mathrm{mg} / \mathrm{kg}[\mathrm{F}(3,54)=$ $4,16, \mathrm{p}<0,01]$ et l'activité locomotrice à la dose de $1 \mathrm{mg} / \mathrm{kg}[\mathrm{F}(3,54)=2,78, \mathrm{p}<0,05]$. La 4-phényl-1,5-benzodiazépin-2-one a aussi 


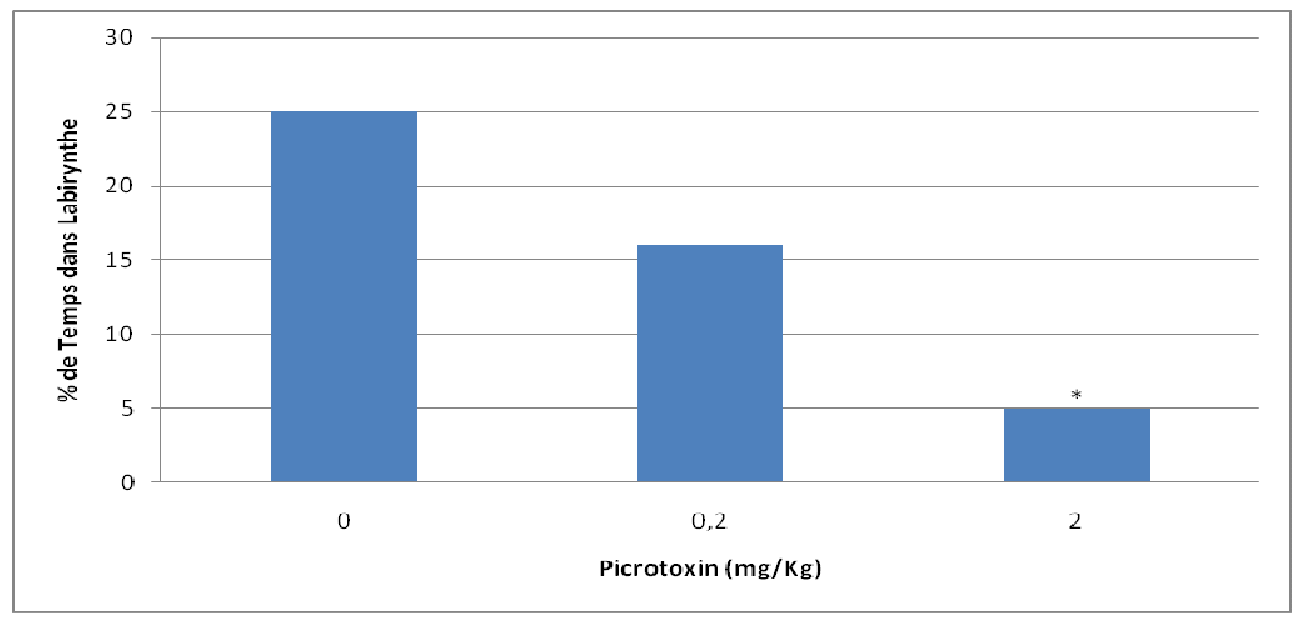

Figure 2 : Effets de picrotoxin sur le temps passé dans les bras ouverts par des souris.

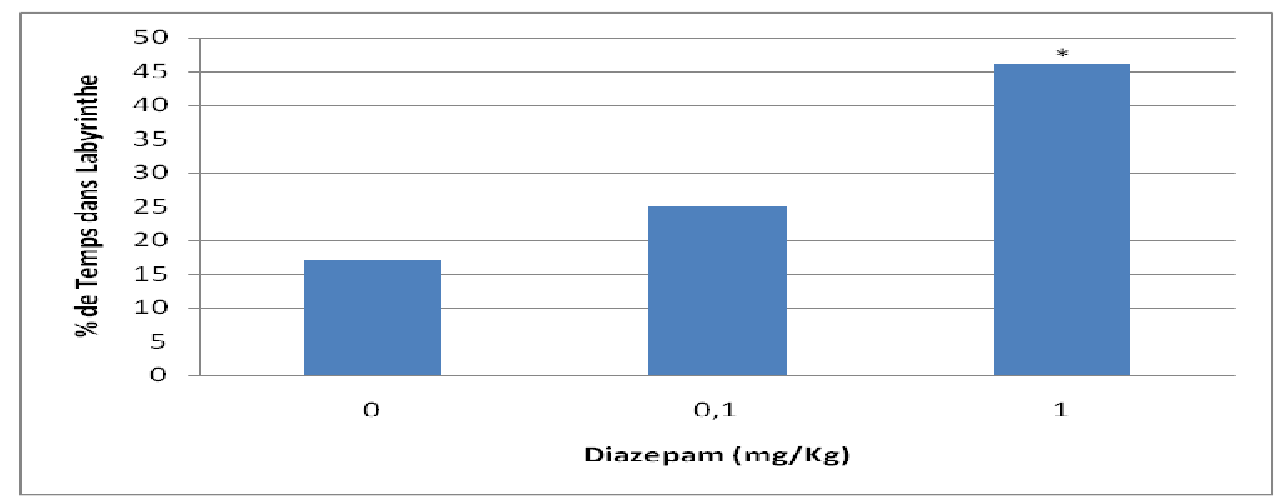

Figure 3 : Effets du diazépam sur le temps passé dans les bras ouverts par des souris.

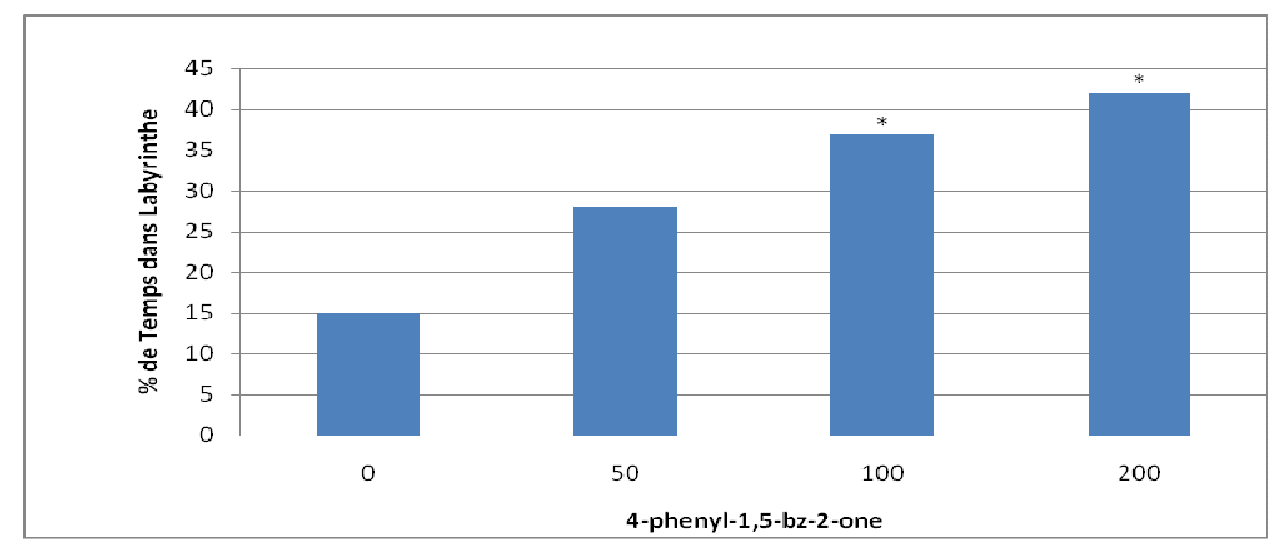

Figure 4: Effets de la 4-phényl-1,5-benzodiazépin-2-one sur le temps passé dans les bras ouverts par des souris. Les résultats sont présentés sous forme des moyennes \pm Ecart types; $\mathrm{N}=15$ souris par groupe. $\mathrm{P}<0,05$ par rapport au véhicule (Test Student-Newman-Keuls ). 
Tableau 2: Effets de picrotoxin, du diazépam et de la 4-phényl-1,5-benzodiazépine sur le comportement des souris dans le test de planche à trous.

\begin{tabular}{lcc}
\hline & Nombre de trous explorés & Activité locomotrice \\
\hline Véhicule & $19,3 \pm 2,6$ & $110,9 \pm 10,2$ \\
Picrotoxin $(0,2 \mathrm{mg} / \mathrm{Kg})$ & $19,8 \pm 2,8$ & $98,9 \pm 8,4$ \\
Picrotoxin $(2 \mathrm{mg} / \mathrm{Kg})$ & $7 \pm 1,4^{*}$ & $69 \pm 0,9^{*}$ \\
Diazepam $(0,1 \mathrm{mg} / \mathrm{Kg})$ & $13 \pm 2,1$ & $112,5 \pm 10$ \\
Diazepam $(1 \mathrm{mg} / \mathrm{Kg})$ & $9,1 \pm 2,2$ & $115,5 \pm 16,8^{*}$ \\
4-PBZ $(50 \mathrm{mg} / \mathrm{Kg})$ & $17,6 \pm 2,5$ & $111,4 \pm 9,5$ \\
4-PBZ $(100 \mathrm{mg} / \mathrm{Kg})$ & $12,5 \pm 1,2$ & $102,2 \pm 6,6$ \\
4-PBZ $(200 \mathrm{mg} / \mathrm{Kg})$ & $9,3 \pm 1,3^{*}$ & $99,7 \pm 7,2$ \\
\hline
\end{tabular}

Les résultats sont présentés sous forme des moyennes \pm Ecart types; $\mathrm{N}=14$-18 souris par groupe. $* \mathrm{P}<0,05$ par rapport au véhicule (Test de Student-Newman-Keuls).

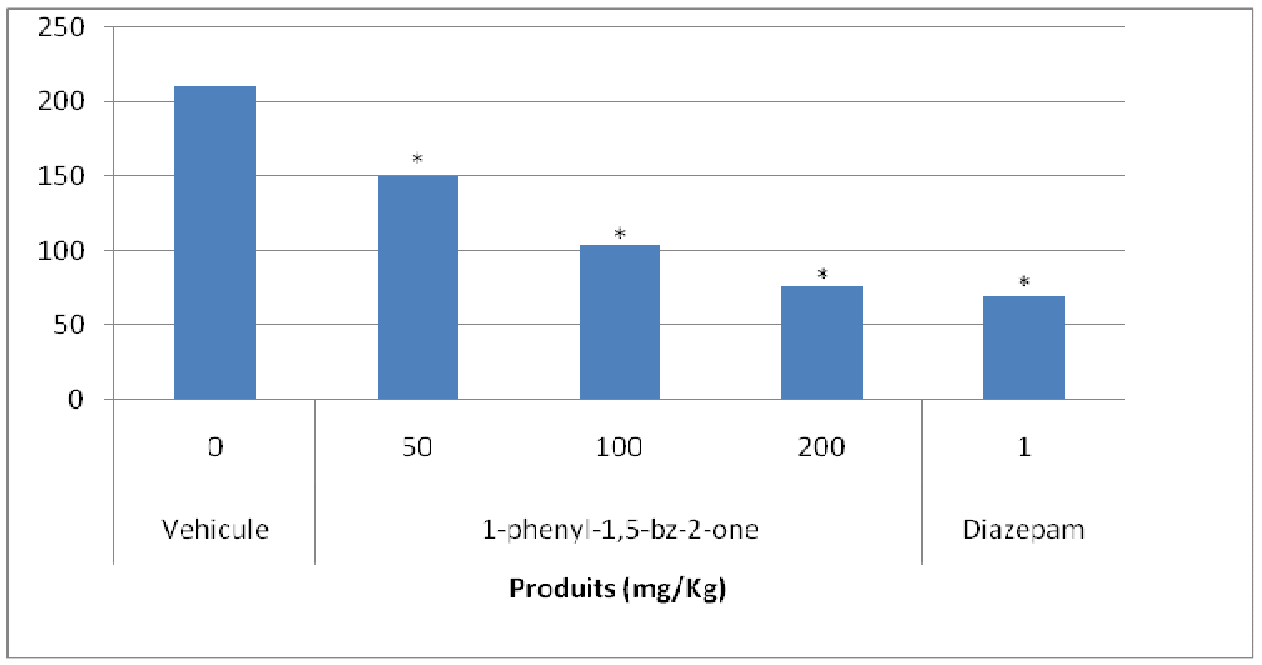

Figure 5: Comportement des souris sur la tige tournante après administration du véhicule, de la 4-phényl-1,5-benzodiazépin-2-one et le diazépam. Les résultats sont présentés en seconde (moyennes \pm Ecart types), $\mathrm{N}=15$ souris par groupe. $* \mathrm{P}<0,001$ par rapport au véhicule (Test de Student-Newman-Keuls).

provoqué une réduction du nombre de trous explorés par les souris $[\mathrm{F}(5,98)=2,57$, $\mathrm{p}<0,05]$, ainsi que leur activité locomotrice (Tableau 2).

Test de la tige tournante : L'analyse des données de ce test révèle que le diazépam (1 mg/kg) et la 4-phényl-1,5-benzodiazépin-2one aux différentes doses $(50,100$ et 200 $\mathrm{mg} / \mathrm{kg}$ ) ont modifié de manière importante le comportement des animaux par rapport au véhicule $[\mathrm{F}(4,70)=7,6 \mathrm{p}<0,001)]$ (Figure 5).

\section{DISCUSSION}

L'analyse statistique des données du test de labyrinthe a montré que la picrotoxin a diminué le nombre d'entrées et le temps passé dans les bras ouverts par les animaux $[\mathrm{F}(3,59)$ $=8,32, \mathrm{p}<0,001$, Figure 2], confirmant ainsi 
les données de la littérature qui suggèrent un rôle anxiogenique de ce produit. Par contre, le diazépam a entraîné une augmentation du nombre d'entrées et du temps passé dans les bras ouverts $[\mathrm{F}(3,53)=3,78, \mathrm{P}<0,05$, Figure 3]. Considéré comme un des tests spécifiques pour l'étude de l'anxiété (Cruz et al., 1994; Hogg, 1996), ce dernier est aussi validé pour être utilisé avec la souris, et il a été prouvé que ce test est sensible pour les produits qui agissent par l'intermédiaire du récepteur GABA-A (Hogg, 1996; Rodgers et al., 1997). Raisons pour lesquelles, nous l'avons choisi pour étudier le potentiel anxiolytique de la 4phényl-1,5-benzodiazépin-2-one. Sa validité dans notre étude a été soutenue par l'effet que la picrotoxin anxiogenique typique et le diazépam, un anxiolytique classique, ont sensiblement diminué et augmenté le nombre d'entrées et le temps passé dans les bras ouverts par les souris (Figures 2 et 3). La 4phényl-1,5-benzodiazépin-2-one, tout comme le diazépam, a aussi entraîné une augmentation du nombre d'entrées et du temps passé dans les bras ouverts par les souris (Figure 4). Ces résultats suggèrent que la 4-phényl-1,5-benzodiazépin-2-one a un effet anxiolytique.

$\mathrm{Au}$ regard des résultats du test de planche à trous nous observons que la picrotoxin $(2 \mathrm{mg} / \mathrm{kg})$ a considérablement diminué le nombre de trous explorés et le nombre des zones traversées par les souris avec toutes leurs pattes. La 4-phényl-1,5benzodiazépin-2-one a aussi réduit de manière significative le nombre des trous explorés, ainsi que l'activité locomotrice des animaux, ce qui suggère l'existence d'un effet sédatif, pouvant permettre de confirmer l'activité anxiolytique de la molécule observée lors du test de labyrinthe. Cet effet a été aussi observé lors du test de la tige tournante, la dose de $200 \mathrm{mg} / \mathrm{kg}$ a démontré une activité similaire à celle du diazépam, alors que la dose de 50 $\mathrm{mg} / \mathrm{kg}$ a démontré une très faible activité (Figure 5).

Ces résultats démontrent que la 4phényl-1,5-benzodiazépin-2-one présente une activité anxiolytique comparable à celle du diazépam à différentes doses utilisées, même si cette activité est plus remarquable à la doses $200 \mathrm{mg} / \mathrm{kg}$, d'où la nécessité de synthétiser des dérivés de cette molécule avec différents substituants pouvant améliorer l'activité de la molécule de base (la 4-phényl-1,5benzodiazépin-2-one).

\section{Conclusion}

Le profil de comportement induit par la 4-phényl-1,5-benzodiazépin-2-one sur base des trois tests comportementaux utilisés dans cette étude, est similaire à celui induit par le diazépam, néanmoins la 4-phényl-1,5benzodiazépin-2-one contrairement au diazépam, n'a pas réussi à accroître l'activité locomotrice des animaux. Ces résultats démontrent que la 4-phényl-1,5benzodiazépin-2-one possède une activité anxiolytique, cette activité est franche à la dose de $200 \mathrm{mg} / \mathrm{kg}$.

\section{REMERCIEMENTS}

Ce travail a été soutenu par l'Agence Marocaine de Coopération Internationale (AMCI) que nous remercions vivement.

\section{REFERENCES}

Anonyme. 1996. Guide pour les Soins et l'Utilisation des Animaux de Laboratoire, Institute of Laboratory Animal Resources. National Academy Press: Washington D.C.

Beaulieu P. 2006. Pharmacologie de la Douleur. Les Presses de l'Université de Montréal: Montréal.

Broustail M. 1967. La Souris de Laboratoire et son Elevage ( $3^{\text {éme }}$ Edn). Vigot Frères : Paris.

Cherrah Y, Alaoui K, Faouzi MY A. 2007. Travaux pratiques de pharmacologie. $2^{\text {eme }}$ année de pharmacie, Faculté de Médecine et Pharmacie de Rabat.

Cruz AP, Frei F, Graeff FG. 1994. Ethopharmacological analysis of rat behavior on the elevated plus-maze. Pharmacology, Biochemistry and Behavior, 49: 171-176.

De Sarro GB, Rotiroti D, Gratteri S, Sinopoli S, Juliano M, De Sarro A. 1992. Tolerance to anticonvulsant effects of clobazam, diazepam, and clonazepam in genetically epilepsy prone rats. Advances in Biochemical Psychopharmacology, 47: 249-254.

Essassi EM, Fifani J. 1987. Synthèse de la 4acétonylidène-1,5 benzodiazépin-2-one. J. Bull. Soc. Chim. Belg., 96: 63-69. 
Ernest D, Olfert DVM, Brenda M, Cross DVM, Ann McWilliam A. 1993. Manuel sur le Soin et l'Utilisation des Animaux d'Expérimentation (Vol. 1). Conseil Canadien de Protection des Animaux.

Fernandes C, File SE, Berry D. 1996. Evidence against oppositional and pharmacokinetic mechanisms of tolerance to diazepam's sedative effects. Brain Research, 734: 236-242.

Ferreri M. 2004. Anxiété, Anxiolytiques et Troubles Cognitifs, L'Anneau Pelvien (Ostéopathie). Masson: Paris.

Graham L. Patrick, Depovere P. 2002. Chimie pharmaceutique. De Boeck Université.

Hogg S. 1996. A review of the validity and variability of the elevated plus-maze as an animal model of anxiety. Pharmacology, Biochemistry and Behavior, 54: 21-30.

Kirkiacharian.S. 1996. Guide de Chimie Thérapeutique. Ellipses: Paris.

Laroche M-J, Rousselet F. 1990. Les Animaux du Laboratoire: Ethique et Bonnes Pratiques. Masson : Paris.

Leboyer M. 2005. Troubles bipolaires: pratiques, recherches et perspectives: pratiques, recherches et perspectives, John Libbey Eurotext.

Lister RG. 1987. The use of plus-maze to measure anxiety in the mouse. Psychopharmacology, 92: 180-185.

Perlemuter L. 2008. Guide de Thérapeutique. Masson: Paris; 2145p.

Remy C. 1994. Clobazam in the treatment of epilepsy: a review of the literature. Epilepsia, 35: S88-S91.

Rodgers RJ, Johnson NJT, Carr J, Hodgson TP. 1997. Resistance of experimentallyinduced changes in murine plus-maze behaviour to altered retest conditions. Behavioural Brain Research, 86: 71-77.

Tosti A, Fleckman P, Scher R, Ralph Daniel C. 2007. Onychologie Diagnostic, Traitement, Chirurgie. Masson: Paris.
Tsuda M, Suzuki T, Misawa M, Nagase H. 1996. Involvement of the opioid system in the anxiolytic effect of diazepam in mice. European Journal of Pharmacology, 307: 7-14.

Van Gerven JM, Roncari G, Schoemaker RC, Massarella J, Keesmaat P, Kooyman H, Heizmann P, Zell M, Cohen AF, Dingemanse J. 1997. Integrated pharmacokinetics and pharmacodynamics of Ro 48-8684, a new benzodiazepine, in comparison with midazolam during first administration to healthy male subjects. British Journal of Clinical Pharmacology, 44: 487-493.

Wildlocher D. 2002. Dépression et anxiété. Revue française de Psychanalyse, 66: 409-422.

Wolfman C, Viola H, Paladini A, Dajas F, Medina JH. 1994. Possible anxiolytic effects of chrysin, a central benzodiazepine receptor ligand isolated from Passiflora coerulae. Pharmacology, Biochemistry and Behavior, 47: 1-4.

Yasumatsu H, Morimoto Y, Yamamoto Y, Takehara S, Fukuda T, Nakao T, Setoguchi M. 1994. The pharmacological properties of Y-23684, a benzodiazepine receptor partial agonist. British Journal of Pharmacology, 111: 1170-1178.

Zangrossi Jr H, Viana MB, Graeff FG. 1999. Anxiolytic effect of intra-amygdala injection of midazolam and 8-hydroxy-2(di-n-propylamino)-tetralin in the elevated T-maze. European Journal of Pharmacology, 369: 267-270.

Zellou A, Cherrah Y, Essassi EM, Hassar M. 1998. Synthèse et étude pharmacologique des 1,5-benzodiazepine-2diones et leurs dérivés alkylés. Ann. Pharm. Fr., 58(4): 169-174. 